\title{
How to spend and invest retirement savings.
}

\author{
Aparna Gupta and Walter Murray* \\ Management Science and Engineering, Stanford University, Stanford CA 94305
}

\begin{abstract}
The question of post-retirement optimal consumption and investment of retirement savings is addressed. This problem has received considerably less attention than that of how to invest for retirement. With the increase in life span and an increase in private pension funds, a retiree has considerable flexibility in both how to consume and how to continue to invest their retirement funds. Our interest is in developing a platform that allows a wide variety of behavioural aspects to be modeled and also enables explicit constraints to be imposed. To enable the flexibility we seek it is necessary to model the problem as a large-scale nonlinearly constrained optimization problem, which is solved using a sequential quadratic programming algorithm. Fortunately, modern optimization methods are now sufficiently powerful as to enable solving such problems. A key point is that, though the problems are large, they have a rich structure.

Problems in this class have been addressed assuming that an investor is rational in the sense that when making financial decisions the preference relation of the investor satisfies all

\footnotetext{
${ }^{*}$ Work supported by the National Science Foundation grant CCR-9988205 and Office of Naval Research
} grant N00014-96-1-0274
\end{abstract}


the axioms of choice. Research in behavioural science indicates that not all financial decisions of an average person satisfy the axioms of choice. The algorithm we propose enables the problem to be solved for a user-specified utility function that does not satisfy all the axioms of choice. 


\section{Introduction}

The problem of how an investor should invest for acquiring adequate retirement savings is viewed as an important problem and has been the subject of considerable research $[23,18$, 19, 3, 29]. An associated problem that has not received as much attention, but in our view is one of equal significance, is the problem of optimal spending and investing of retirement funds during retirement. With life expectancy increasing, the years spent in retirement are comparable with number of pre-retirement investment years.

In the traditional framework for retirement planning usually three components are considered and modeled. An income stream one receives or expects to receive, what part of that income one decides to consume or spend immediately, and finally what part of it one saves and invests for future consumptions. In practice most people in the US set the percentage of their income they invest in retirement only once, which is typically at a level equal to or lower than the maximum allowed tax-deductible contribution. In contrast, consumptions after retirement have considerably more flexibility. In our view the problem of saving for retirement and that of consuming retirement savings after retirement have very different characteristics, and there is merit in modeling them differently. Moreover, a retiree is more motivated to take an interest in how best to invest since the consequences are immediate. Another key difference is the capacity to recover from a poor outcome. In pre-retirement a person usually has a greater capacity both to improve their income stream (by working harder) and reduce their consumption (greater discretionary spending).

In developing a platform we wanted to address a number of concerns. In particular we have concerns that current platforms are too restrictive and there is a lack of transparency. 
As an example of the latter suppose a user does not wish his wealth to fall below $\$ x$. One could create a utility function that becomes infinitely negative when wealth approaches $\$ x$. An average user would not understand the properties of such a function. A more transparent approach is to have a constraint $W>=x$. Of course 'transparency' is subjective; our task is to have a flexible platform that can cope with a wide variety of constraints and utility functions. One way of achieving this is to allow quantities such as wealth to appear directly in the model as a variable. One consequence of our approach is the number of variables may become very large. However, modern algorithms $[7,8]$ are more dependent on structure than size. It will be seen that increasing the number of variables improves structure. Furthermore, the efficiency of such algorithms depends more on the degrees of freedom in the problem rather than the number of variables. The degrees of freedom remain unchanged when additional variables are introduced, since an identical number of additional constraints are also introduced.

In defining the utility function, assumptions are usually made about the planner's preferences for consumption, the assets available for investment and rules for their price evolution. Heretofore, problems in this class have been addressed assuming that an investor is rational in the sense that when making financial decisions the preference relation of the investor satisfies all the axioms of choice. Research in behavioural science indicates that not all financial decisions of an average person satisfy the axioms of choice $[13,27,14]$. The term "rational" is somewhat of a loaded term since it implies if an individual does not satisfy the axioms of choice, they are being irrational. Hoewever, this is a technical term in this context and as was pointed out in $[11,10]$, many investors have sound reasons for not satisfying the 
axioms of choice. The framework we present enables behavioural aspects to be included in the model by using a behavioural utility and/or adding behavioural constraints. The traditional utilities can also be solved with this approach. For illustrative purposes, assets available for investment are categorized into two major classes, risky and riskfree. Further refinements are possible. For expository purposes we model the returns of the assets using the binomial-tree model (Sharpe [24]).

\section{Modeling the Problem}

\subsection{Normative versus Descriptive Choice}

An individual faced with a decision chooses from a set of alternatives. The decision-maker weighs the merits and de-merits of each option among all the alternatives and attempts to select the option that suits him best. This usually requires a precise definition of "best." In order to perform an analysis of this decision-making process, it is necessary to define a preference relation. Furthermore, the preference relation is assumed to satisfy certain axioms of choice that impose a consistency and regularity in the decisions governed by the relation. (See Gupta and Murray [11], Varian [28], MasColell [17] for more details.) A decision-maker who satisfies these axioms is said to be economically rational. It is possible to represent the preferences in terms of a utility function, which is a map from the set of alternatives to the real line. A consequence of the axioms being satisfied is that the corresponding utility function is well-behaved.

In practical terms, however, the imposition of these axioms has enormous implications and it may be a difficult proposition for an average decision-maker to satisfy all the axioms 
of choice all the time. Moreover, many people do not view adhering to the axioms of choice as being desirable since they conflict with their intuitive preferences on which they make decisions. Following this thought many behavioural scientists and economists have attempted to understand the decision-making processes among ordinary people through experiments and studies of various kind (see Wright [30], Bell [1]). These attempts also try to quantify the choice-making process, but try to be parsimonious in their assumptions. They constitute what is called the descriptive theory of choice. The five major phenomena of choice that violate the standard model of normative theory are listed as : framing effects, nonlinear preferences, source dependence, risk seeking and loss aversion. These have been confirmed in a number of experiments, with both real and hypothetical payoff (see Gupta and Murray [11], Tversky \& Kahnemann [27, 26], Tversky [25] for more details).

Behavioral finance is a fast-growing area of research. In this framework researchers have made an attempt to understand and explain the investment style and preferences of individual investors and other market characteristics from a descriptive stand point. It is hoped that these descriptive studies will help bridging the gap between the theories developed on the rationality hypothesis and what is observed in practice. Bridging this gap will help experts in providing better advice and 'educate' people to make better decisions.

\subsection{The Consumption Problem}

The consumption problem is a problem involving choice. It refers to the problem of finding the optimal consumption of retirement savings after retirement. An accompanying optimal investment strategy to support the consumption also needs to be computed. As it was pointed out earlier, most researchers have studied the consumption problem in conjunction with the 
saving and investment problem. We choose to study the consumption problem separately, since the two stages of retirement planning, namely the time in life when one plans his retirement and the time when one is retired, have very different characteristics, concerns and preferences. A key difference between pre-retirement and post-retirement decisions is that the post-retirement decisions have an immediate impact. Moreover, a retiree may need to respond to rapidly changing circumstances such as a deterioration in health. One may also expect personal utilities to vary more widely since there is now a consumption aspect.

Most would agree that to achieve retirement goals requires a well-defined plan. The most important considerations for this planning would be:

- when one retires or the retirement age,

- expected lifestyle and related expenses after retirement,

- what is one's life expectancy, or general health condition,

- what different sources of income one has after retirement, such as social security, annuities, personal savings

- marital status,

- and whether or not there is a bequest motive.

Clearly, a lot depends on how early and how well an individual plans for his retirement and accumulates the retirement capital. We assume that the planner has done their best to accumulate their retirement fund. With this assumption, we address how the individual should consume the fund after retirement and how what is not consumed in a given period 
should be invested in order to keep the best flow of income during the retirement years. The investment choice set will typically contain elements such as how much to invest, what to invest in, how should one invest in order to have maximum tax benefits, etc. Income from sources such as annuities or social security are treated as exogenous to the problem set-up, and only affect the lower bound on the consumption variables.

Modeling life expectancy is a critical aspect of the problem. A related issue is a retiree's health. This may impact both consumption due to health care costs and the retiree's ability to enjoy life. The uncertainty related with health is at the highest during retirement, and modeling of the problem should take this into account. Most other considerations listed above for retirement planning can usually be incorporated as parameters of the model, for instance, marital status can be accommodated by considering larger consumption volumes, similar adjustment could be made for life-style related expenses.

The problem is mathematically formulated as

$$
\max _{X_{t}, C_{t}, t=0, T-1} \sum_{t=0}^{T} E\left[U_{t}\left(W_{t}, C_{t}\right)\right],
$$

where $C_{t}$ and $X_{t}$ are consumption and investment decisions over time, respectively. The planning horizon, $T$ for this problem is not known with certainty. The last term in the objective, $U_{T}\left(W_{T}, C_{T}\right)$, will represent a bequest preference.

\subsection{Overview of other Approaches}

Most work on this problem has been in an integrated framework along with the investment problem. In this section we give a brief overview of these approaches. Bodie, Merton and Samuelson [2] consider a life-cycle model with an optimal investment choice, choice of labor 
and consumption over the life-time of the planner. The problem is posed as an optimal control problem in continuous time and solved by assuming that wages are perfectly hedged. Relations are drawn between the optimal investment choice and human capital of the planner over the planning horizon. Campbell and Viceira [3] consider an infinitely lived investor with Epstein-Zin-Weil utility (a recursive generalization of the power utility) and solve the investor's optimal consumption/investment problem. They replace the first-order conditions (Euler's equation) and budget constraints of the problem with approximate equations and explore the analytical solution for the approximate problem. They analyse trends such as the optimal growth rate of consumption, optimal consumption-wealth ratio, etc. Viceira [29] extended Campbell-Viceira approach by including an undiversifiable labor income process in the problem. Investors are infinitely lived but have a certain probability, $\pi_{r}$, of transition to a retired state. After reaching a retired state investors stay retired thereafter. Power utility for consumption is assumed, the solution technique is similar to that given by Campbell-Viceira, and an analysis is done on the effect of retirement on the portfolio choice.

Several researchers have performed empirical analyses to understand retirement behaviour among individuals. Hurd and McGarry [12] study the evolution of subjective survival probabilities and their ability to predict actual mortality. They found that subjective probabilities reasonably predict actual survival. In Rust [22] a dynamic programming based model for retirement behaviour is developed and solved using a nested fixed-point algorithm. At each time step the worker chooses how much to consume and whether to work full-time, part-time, or exit the labor force. The objective is to maximize expected discounted utility over the worker's remaining life-time. Lumsdaine, Stock and Wise [16] study the predictive perfor- 
mance of three models of retirement behaviour - "option value," dynamic programming, and probit. They advocate the use of a simpler model with good predictability, and find that in this sense the "option value" model does better than the dynamic programming one.

\subsection{Asset-price dynamics - Binomial Tree Set-up}

Future consumptions depend on the overall retirement savings and, given the length of retirement, critically depends on how well the retirement fund gets invested. Investment growth, in turn, is driven by what we invest in, how long we invest, what return we earn and how much of that return we surrender to transaction costs and to taxes. Individual investors usually have a great deal of control over every factor above, except one, the asset returns. The returns we get on our investments are uncertain. Past performances may be indicative of the future performance, but in no way are fully predictive. This fact makes modeling the asset-return dynamics particularly challenging. For illustrative purposes we use the binomial-tree model (see Gupta and Murray [11], Luenberger [15] and Ross [20] for details). In this discrete-time, discrete-space asset-return dynamics model, asset-returns can transition to two possible states at each point of time. We call one of the states an "up" and the other a "down." The "up" refers to a return greater than 1, or in other words, the price of the asset goes up, and the "down" refers to the return being less than 1. Although it may look simplistic, this model has enjoyed enormous success due to its ease of use $[24,4,21]$ and yet its capacity to capture important characteristics of the problem.

Throughout this work we consider two asset-classes, a risky one and a riskfree one. Again we emphasize that this is done for illustrative purposes. We are not advocating that the binomial-tree model is to be preferred. A simple extension is to have a greater refinement in 


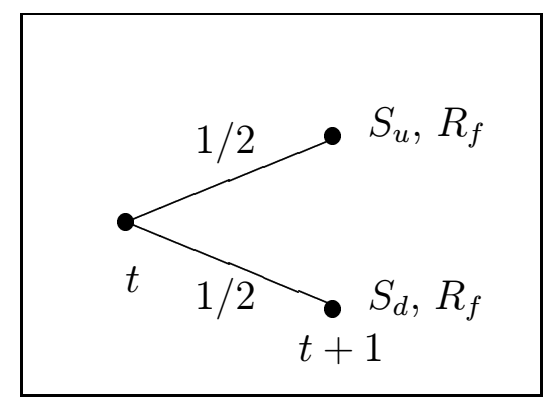

Figure 1: One period transition rule for binomial-tree model

the assets available for investment, such as stocks, bonds and money market account (cash). At each point of time the risky asset's return goes up with a probability $p=1 / 2$ and down with probability $1-p=1 / 2$, whereas the return of the riskfree asset remains constant, hence the name "riskfree." Similar to refinements in the asset classes, several refinements in the asset return dyanmics may also be introduced. We denote the return of the risky asset on the "up" by $S_{u}$ and $S_{d}$ on the "down." Let the return of the riskfree asset be $R_{f}$. (The parameter values chosen are as follow, $S_{u}=1.26, S_{d}=0.96, R_{f}=1.05$, to match the mean and standard deviation of long-term yearly returns of the US stock market proxy and risk premium.) If one invests $W_{s}$ dollars in the risky asset and $W_{b}$ in the riskfree asset in a period, the wealth one would have in the next period will be $W_{s} S_{u}+W_{b} R_{f}$ if an "up" occurs and $W_{s} S_{d}+W_{b} R_{f}$ if a "down" occurs. Figure 1 gives a pictorial description of the binomial-tree model.

\subsection{Optimal Consumption \& Asset Allocation Problem}

With the binomial-tree model described in the previous section the optimal consumption and asset allocation problem can be formulated as follows. Given a time-horizon for the planning, 
$T$, for each time point, $t$ lying in $\{0,1, \ldots, T-1\}$, a decision regarding the amount of wealth to withdraw from the fund for consumption and the amount of wealth to invest in the two asset-classes needs to be made. Assuming the investor knows the state of the world at $t=0$, there are $2^{T}-1$ number of nodes between $t=0$ and $t=T-1$, each representing two decision variables, how much of wealth to consume and how much to invest in the risky asset and the riskfree asset. The nodes are ordered as indicated in figure 2. Let the investment decision variable be denoted by $X_{t, n}$, where $t$ is the time period and $n$ is the node in the ordering. The consumption decision variables are denoted by $\left\{C_{t, n}, t=0 . . T, n=1 . .2^{T}-1\right\}=\mathrm{C}$. Our convention is that the planner takes out some money from the fund at the beginning of each period for consumption during the period and invests the remaining wealth in the two asset-classes. This implies that at $t=0$, which is the time the planner retires, given the total wealth in the retirement fund is $W_{0}$, the planner withdraws $C_{0,1}$ dollars for consumption in the first period and invests the remaining wealth in the two asset classes. Continuing in this manner, there will be an amount of wealth associated with each node in the tree, denoting the wealth remaining in the retirement fund at that node. We represent these wealth levels by $W_{t, n}$, following the convention for the two decision variables. Therefore, the relationship between the decision variables $C_{t, n}, X_{t, n}$ and the state variables $W_{t, n}, S_{u}, S_{d}$ and $R_{f}$ can be written as follows:

$$
\begin{gathered}
W_{t+1, n_{2}}=X_{t, n_{1}}\left(W_{t, n_{1}}-C_{t, n_{1}}\right) S_{u}+\left(1-X_{t, n_{1}}\right)\left(W_{t, n_{1}}-C_{t, n_{1}}\right) R_{f} \\
W_{t+1, n_{3}}=X_{t, n_{1}}\left(W_{t, n_{1}}-C_{t, n_{1}}\right) S_{d}+\left(1-X_{t, n_{1}}\right)\left(W_{t, n_{1}}-C_{t, n_{1}}\right) R_{f}, t=0 . . T-1, n_{1}=1 . .2^{T}
\end{gathered}
$$

where (1) is for an "up" and (2) for a "down." $\left\{X_{t, n}, t=0 . . T, n=1 . .2^{T}-1\right\}=\mathrm{X}$ are taken to be the fraction of ones wealth at time $t$ and node $n$ one invests in the risky asset. 


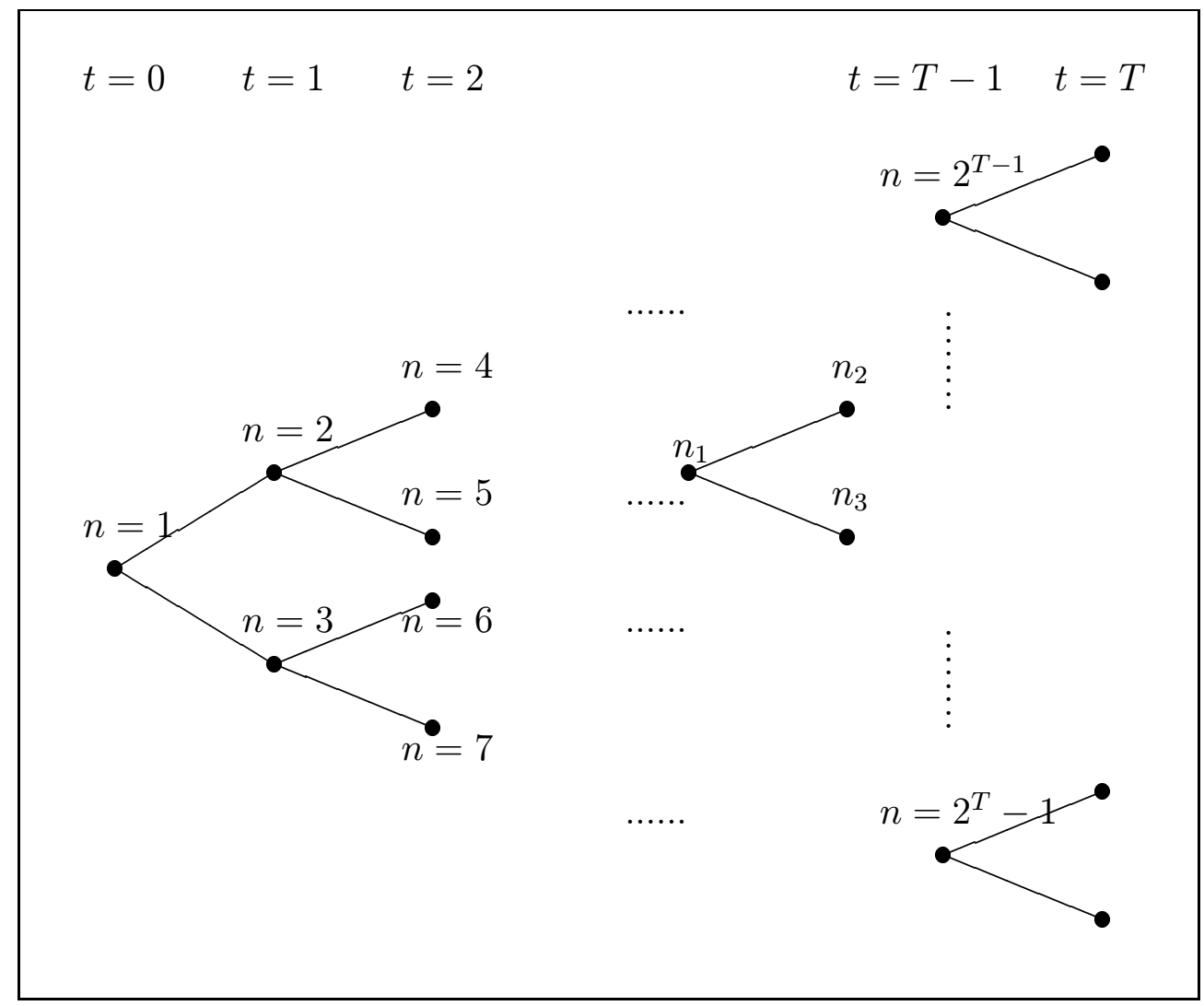

Figure 2: Ordering of nodes in the binomial tree

Therefore, $\left(1-X_{t, n}\right)$ is the fraction of remaining wealth invested in the riskfree asset at time $t$ and node $n$. The relationship between the nodes $n_{1}, n_{2}$ and $n_{3}$ is indicated in figure 2 .

The planner derives utility from consumption, hence the objective of the problem will be to maximize the expected utility of consumption. In this model of the problem we take the planning horizon as the life-expectancy; the true planning horizon of the problem is uncertain. This aspect is discussed further in Section 2.6. A typical retiree is likely to have a preference for consumption that is time dependent. For example, given the uncertainty of remaining alive, the planner may prefer to spend money earlier rather than later. Moreover, he may well be able to travel and indulge in costly activities that become impossible later. Conversely, he may expect health-related costs to rise with age and may wish to plan for 
such expenditure. To illustrate the role of time in altering preferences we introduce a time preference parameter, $\delta$. Utility for consumption at time, $t$ is taken to be $\delta^{t} U\left(C_{t}\right)$, where $\delta$ lies in the interval $(0,1)$. As a result, utility from consumption in periods farther out in the planning period get incrementally discounted. The inclusion of $\delta$ in this fashion is for illustrative purposes. An individual could vary $\delta$ with time and may designate specific periods for preferred spending. Besides the utility from consumption, there may also be a bequest term included in the objective, which is the utility for wealth remaining at the planning horizon. Under these assumptions the optimal consumption and asset allocation problem may be written as:

$$
\begin{gathered}
\max _{X, C} \sum_{t=0}^{T-1} E_{0}\left[\delta^{t} U\left(C_{t, .}\right)\right]+E_{0}\left[\delta^{T} U\left(W_{T, .}\right)\right] \\
W_{T, m_{1}}=X_{T-1, m}\left(W_{T-1, m}-C_{T-1, m}\right) S+\left(1-X_{T-1, m}\right)\left(W_{T-1, m}-C_{T-1, m}\right) R_{f},
\end{gathered}
$$

where $S$ takes the value $S_{u}$ when the transition from $m$ to $m_{1}$ is an "up" and the value $S_{d}$ when the transition is a "down." $U($.$) is the utility for consumption and for wealth at the$ planning horizon, $\delta$ is the time-preference parameter for consumption and $T$ the random planning horizon. In the above objective function we have taken the utility for consumption and wealth to be the same, although these may in some cases be different. For instance, if there is no bequest motive, there may be a dis-utility for a positive wealth remaining at the planning horizon. Corresponding changes to the objective are easy to make.

In the above optimization problem, we also impose bounds on the decision variables. The problem is formulated so that short selling is not permitted, i.e. borrowing of assets is not allowed. This restriction translates to bounds on the investment decision variables, namely $0 \leq X \leq e$, where $e$ is a vector of 1's. This restriction reflects regulatory constraints on 
positions the planner can take on the asset classes. If some short selling is allowed, it would not seriously alter either the nature of the problem or the method of solution. One set of reasonable bounds on the consumption decisions would be $C \geq 0$, implying that consumption cannot be negative. Although a lower bound of 0 is meaningful for the consumption variables, we believe that a more strict bound may be desirable. The lower bound should reflect the minimum standard of living one requires. With this in view, the lower bound will depend on planning parameters, such as, alternate sources of income, life-style, standard of living, marital status, etc. The bounds are taken to be increasing with time in order to adjust for inflation and an anticipated increase in certain expenses, such as health-related expenses, as one gets older. However, any pattern of bounds may be included in the model without impacting the ease by which it may be solved.

\subsection{Planning Horizon}

It was observed earlier that a modeling aspect of this problem is the planning horizon, $T$. The planning horizon for the retirement saving problem is not an absolutely certain event, but the planner has a considerable amount of control over it. In the consumption problem, however, the planner has little or no control over the planning horizon. This makes it important to capture this characteristic of the problem in the modeling of the problem. One option for dealing with this uncertainty is to use the life-expectancy, $\bar{T}$, for an individual in place of a random value, $T$, and use other modeling techniques to accommodate some of the uncertainty related with $T$. This is a reasonable approach, since the problem is solved on a rolling basis and the solution is implemented only for the initial period. The impact of varying life expectancy can be computed and an individual could, if they wished, take a 
conservative view. When life-expectancy is large, the impact of variation in life expectancy on the initial period will be small. This is particularly true when unequal time periods are used. For example, if the last period is eight years, the fact that the individual lives for 6 or 10 years in this period is not overly significant. Conversely, when life expectancy is small, it is likely to be an accurate estimate. Under such circumstance we can make the time periods, especially the early ones, very small. Since the planning horizon is small, this does not give rise to an unduly large number of periods. Additionally, the bequest term may include a bequest-to-self component to incorporate need for financial support should a person out-live his life-expectancy. The bequest-to-self function may be taken as a decaying function of life-expectancy. An alternative approach is to model the planning horizon with a random variable. However, underlying such an approach is the need for a framework where the optimal solution can be obtained for different realizations of the random variable. Hence the method advocated here may be viewed as a building block for improved modeling of the planning horizon.

The time discount factor, $\delta$, favours early consumption. We propose to have non-uniform time periods for this problem. In the beginning have shorter periods, say quarter or half-year periods and increase the period size towards the end to periods of 4 or 8 years. The discount factor is appropriately adjusted. This implies that for the later, longer periods the utility of consumption is much lower than the utility of consumption in the early periods. Hence early consumption gets even greater preference. Imposing decreasing utility of consumption as time progresses will entail in not having much money left for later consumption, especially when one happens to live much longer than the life-expectancy. This is where the lower 
bounds on consumption variables play an important role. They will make sure that the planner will be able to maintain the minimum living standards desired. The problem is solved on a rolling basis, therefore it is possible to adjust all the parameters of the problem according to changed conditions as one gets older. Life expectancy is adjusted between rolling solves of the problem, hence is modeled accurately.

\section{Method for Solution}

In the previous section we formulated the optimal consumption problem as a nonlinear programming problem in terms of the decision variables, $C$ and $X$. Following the argument in Gupta and Murray [11] regarding the solution method for this problem, we consider the wealth at each node to be a variable in the optimization problem. The wealth values at each node are fully determined once the consumption and investment decisions, $C$ and $X$ respectively, are known. Therefore, we need to include constraints that impose this characteristic of the problem. Equations (1) and (2) become the nonlinear constraints of the problem, where $C_{t, n_{1}}, X_{t, n_{1}}, W_{t, n_{1}}, W_{t+1, n_{2}}$ and $W_{t+1, n_{3}}$ are now variables for all $t, n_{1}, n_{2}, n_{3}$ relevant indices. There are $3\left(2^{T}-1\right)$ variables in the new optimization problem. In the terminology of optimal control, the wealth variables are the state-variables of the problem, whereas $C$ and $X$ are the control variables. In a typical stochastic control problem the number of state variables is much larger than the number of control variables, but in our case they are the same in number, hence treating them both as variables is feasible. (For further details see Gupta and Murray [11].)

In the new formulation our problem is a nonlinearly constrained optimization problem 
with $2^{T}-1$ nonlinear constraints, each involving 4 of the $3\left(2^{T}-1\right)$ variables. Moreover, these constraints are highly structured, they are bilinear, i.e. each term in the left-hand-side of the constraint is a product of two variables. These observations imply that the constraint Jacobian of the problem is very easy to compute, sparse and structured. All these properties can be exploited in an optimization algorithm. Another attractive advantage of this modeling variation is that it is possible to compute the objective gradients with great ease. It is in fact, also possible to compute the Hessian of the objective and constraint function, which enables the Hessian of the Lagrangian to be computed. Like the Jacobian matrix, the Hessian of the Lagrangian is sparse and structured. If the wealth variables and constraints are eliminated, the Hessian of the resulting function is two-third the size, however, it is dense and hard to compute. The gain in efficiency from including wealth as variables greatly out-weighs the loss from an increase in problem size.

There are three major sources of complexity in the above optimization problem. The size of the problem, the nonlinearity of the objective and a possibility of an ill-behaved objective. There are a total of $2^{T}-1$ consumption decision variables, $C, 2^{T}-1$ investment decision variables, and $2^{T}-1$ wealth variables, $W$, all of which appear nonlinearly in the objective. If the expected life-expectancy at retirement is 25 years, we model a 25 years problem using non-uniform time-intervals for our problem periods, and span the 25 years using, say, 20 periods. In a 20 period binomial-tree there are $\left(2^{20}-1=\right) 1,048,575$ nodes, hence twice as many decision variables. The objective may not be so well-behaved since we are interested in solving the problem for a variety of behavioural utilities.

Following Gupta and Murray [11], in seeking a method to solve the consumption problem 
we did not want the method to be too specific to the models currently under consideration. We wanted a powerful general-purpose algorithm that could solve not only our current models, but would allow additional complexity to be added to the model without the need to switch algorithms.

The optimal consumption problem is a nonlinearly constrained optimization problem. The objective has two sets of terms, first terms that evaluate the expected utility of consumption over time, and second those that represent a bequest motive evaluating utility of wealth at the planning horizon. Since the utility function is nonlinear, the objective is nonlinear in the consumption variables. Further, the expression for wealth at the planning horizon is a nonlinear function of wealth levels, consumption and investment decisions at the prior period. Nonlinearity of the utility function further enhances the nonlinearity of utility of wealth term in the objective. The nonlinear constraints arise because the wealth level at each node is treated as a variable in this formulation. Smoothness properties of our objective are largely dependent on the smoothness properties of the utility function $U($.$) , since the$ expression at which the utility is evaluated is a smooth function of the decision variables.

Sequential Quadratic Programming (SQP) methods are considered to be the most robust and efficient algorithms for solving nonlinearly constrained optimization problems. We used the SNOPT [7, 8, 9] software package, a Fortran based package for nonlinear programming from the Systems Optimization Laboratory at Stanford University. SNOPT is a generalpurpose system for solving optimization problems designed to solve problems with a large number of variables and constraints. A key feature of SNOPT and other packages designed to solve large problems is their efficiency as related to the sparseness of the relevant matrices 
such as the Jacobian matrix of the constraint function rather than size. It is for this reason that it is better to increase the size of the problem by having wealth as variables. Likewise, a tree structure, which has many more variables than similar sized lattice is also easier to handle. As pointed out earlier, it is possible to compute the exact Hessian of the objective and constraint function for our problem, which enables the Hessian of the Lagrangian function to be computed. Further, like the Jacobian matrix, the Hessian of the Lagrangian is sparse and highly structured. SNOPT does not use this information, but being able to use these additional features of our problem in the solution procedure would result in a more efficient solution method. It is anticipated that SQP method based on the use of second derivative will be available shortly.

It is worth noting that all methods for obtaining a solution for nonlinear optimization problem are iterative and hence will almost always benefit from starting from a good initial point. In the model and solution method developed here it is not only possible to supply a good point, but also one that is feasible.

\section{Results}

In defining the problem we add lower bounds on the consumption variables for each period. An important piece of information an individual needs to know is given their utility, what is their maximum assured consumption. This consumption serves as a benchmark against which alternative policies can be measured. It also provides a means of bounding poor performance in a manner that is easily understood. The maximum assured consumption is obtained by investing only in riskfree assets. Assuming that there is no bequest requirement, 
the maximum assured consumption is determined by solving the following equations for $C_{f}$.

$$
\begin{aligned}
W_{1} & =\left(W_{0}-C_{f}\right) R_{f} \\
W_{2} & =\left(W_{1}-C_{f} R_{c}\right) R_{f} \\
W_{3} & =\left(W_{2}-C_{f} R_{c}^{2}\right) R_{f} \\
\cdots & \\
W_{T} & =\left(W_{T-1}-C_{f} R_{c}^{T-1}\right) R_{f} \\
W_{T} & =0 .
\end{aligned}
$$

In the above equations it is assumed that consumption in every subsequent period also increases at a rate $R_{c}$ and the risk-free rate is denoted by $R_{f}$. Having determined $C_{f}$, the consumption in time period $k$ is $C_{f} R_{c}^{k}$. Let $\bar{C}$ be a vector such that $\bar{C}_{k}=C_{f} R_{c}^{k}$. We shall refer to $\bar{C}$ as the reference lower bound. The increase in consumption over time, as explained earlier, is justified to not only adjust for inflation, since consumption is taken in nominal terms, but also one expects increased expenditure on health-related needs as one gets older. Similarly, we can find the maximum assured consumption under other assumptions. If the optimal consumption problem is solved for any utility function with the rate of consumption bounded by the reference lower bounds for each time period, then these bounds will all be active. It follows that these lower bounds are the highest feasible level for the lower bound on the consumption variables.

Now consider the case when the planner invests both in the risky and riskfree asset and for simplicity assume $R_{c}=R_{f}$. To enable any optimization we reduce the lower bound on the consumption variables from the reference lower bound by a fraction $\rho$. The new lower bounds are $\rho \bar{C}_{t}$, where $\rho \in(0,1)$. We could of course vary $\rho$ with time without in any way 
increasing the difficulty of solving the problem. The optimal consumption problem is solved with this reduced lower bound. We now apply this approach to a specific utility function. In the framework developed here we can solve the problem for a large class of utility functions, including behavioural utility functions, such as, the S-shaped Prospect theory utility. Results are presented here for a CARA (constant absolute risk-aversion) utility.

\subsection{Negative Exponential utility}

The negative exponential utility is a constant absolute risk aversion utility. Absolute risk aversion, $A(W)$ as defined by Arrow and Pratt [6], is given as

$$
\frac{-U^{\prime \prime}(W)}{U^{\prime}(W)}
$$

for twice continuously differentiable utilities. Absolute risk aversion is a measure for aversion for risk, and for the negative exponential utility is a constant for all levels of wealth. This implies that a planner's appetite for risk is a constant for whatever their level of consumption or wealth. The time-preference parameter, $\delta$ is taken to be 0.9 . A plot of the utility for consumption for an 8 period problem is given in figure 3 .

We solve an 8 period problem with the lower bound relaxed by taking the bound relaxation parameter, $\rho$ equal to 0.8 . In figure 4 a three dimensional plot of the optimal consumption variables is presented. In figure 5 the corresponding optimal investment variables are plotted. Note that at several nodes the optimal investment is to invest $100 \%$ in the risky asset. A 


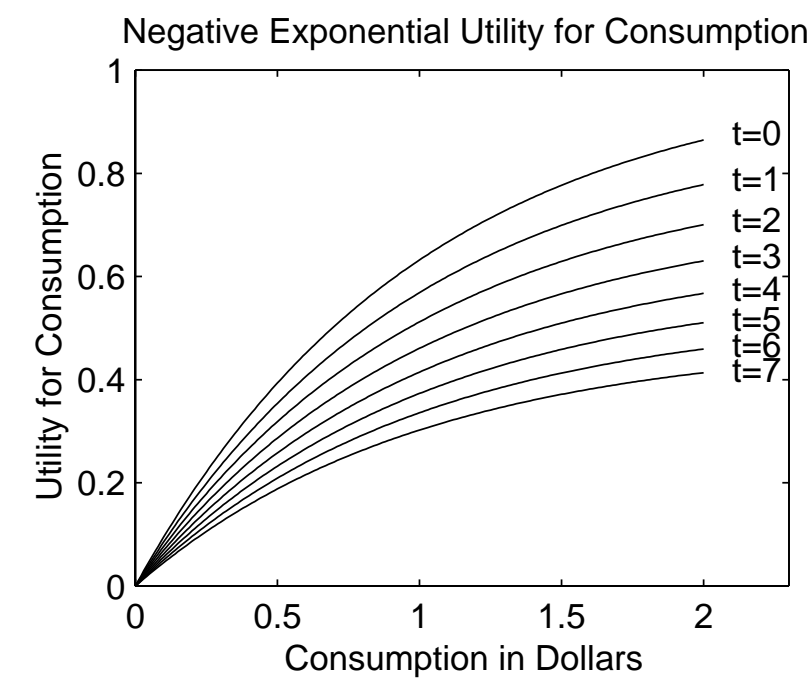

Figure 3:

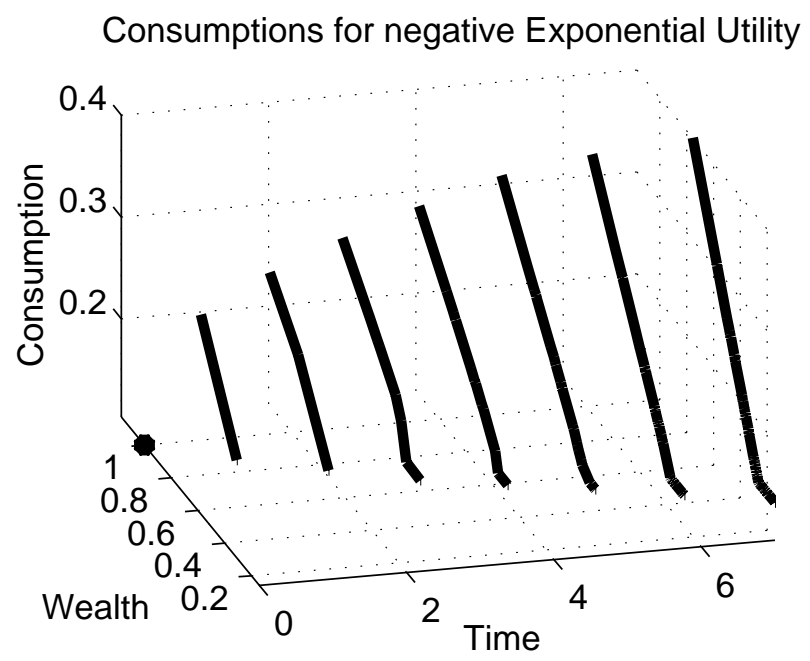

Figure 4: Optimal consumption decisions for an 8 period problem for negative exponential utility 


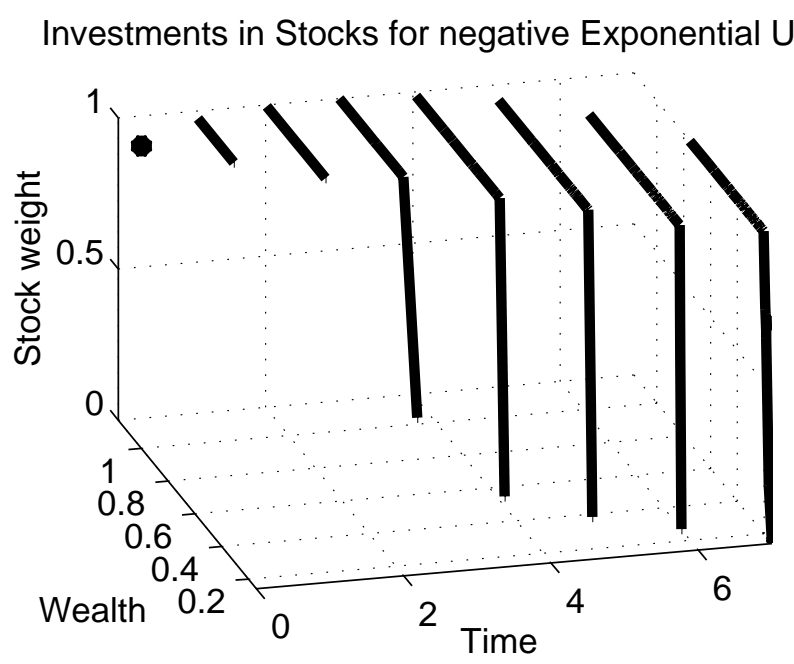

Figure 5: Optimal investment decisions for an 8 period problem for negative exponential utility

question that arises is what is the benefit in terms of consumption levels from taking these risky positions in the investments. One way to measure the "benefit" would be to see the number of nodes in the tree that are above the corresponding reference lower bound level. In figure 6 the percentage of consumption variables for each time period that are not at the problem lower bounds, $\rho \bar{C}_{t}$ and the reference lower bounds $\bar{C}_{t}$ are plotted. The point of this comparison is to see the improvement in consumption from investing in the risky asset. Figure 7 is a plot of minimum, maximum and mean consumption levels for each time period for an 8 period problem.

The results for the optimal consumption problem with the bound relaxation parameter taken as 0.8 indicated that approximately $55 \%$ of the nodes exceed the reference lower bound. 


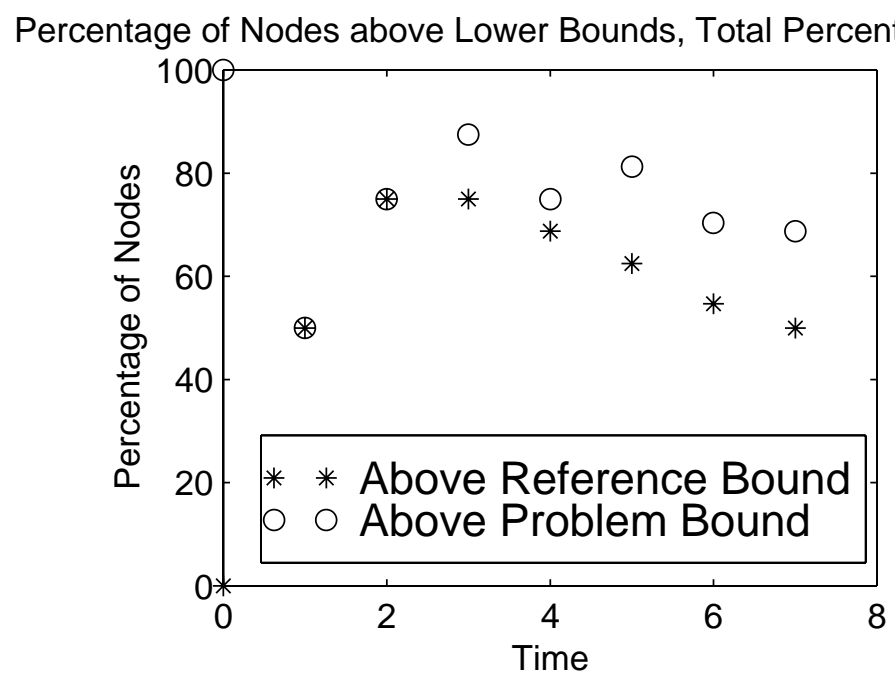

Figure 6: Consumption for each time, $t$ not at the problem lower bound $\rho \bar{C}_{t}$ and not at the reference lower bound $\bar{C}_{t}$

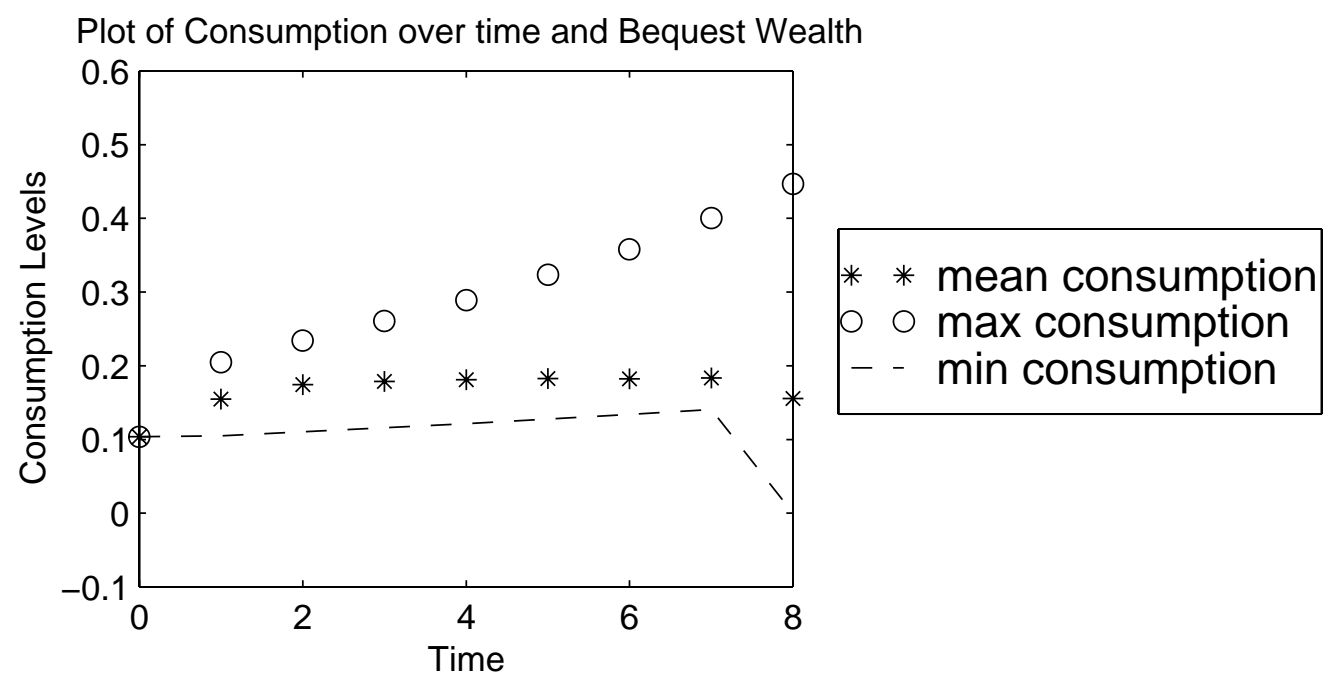

Figure 7: Minimum, Maximum and Mean Consumption level for each time, $t$. 

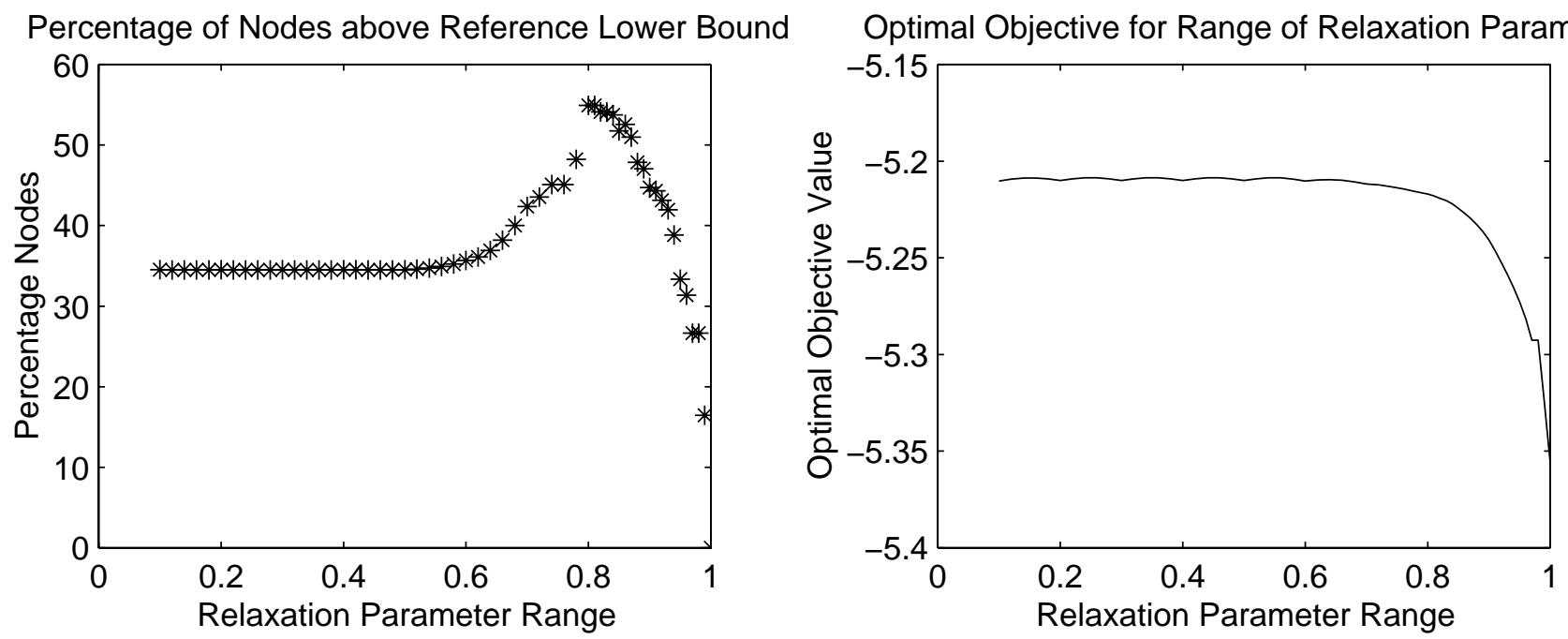

Figure 8: Number of nodes above the reference lower bound $\bar{C}_{t}$ for a range of problem bounds $\rho \bar{C}_{t}, \rho$ ranging from 0.1 to 1.0

We now address the question of how this percentage changes as the relaxation parameter varies in the range 0 to 1 . Figure 8 is a plot of a range of values for the bound relaxation parameter and the corresponding percentage of nodes that are above the reference lower bound. This plot has a very interesting characteristic, for a $\rho$ value around 0.8 the percentage of nodes that exceed the reference lower bound peaks and then asymptotes to approximately a $35 \%$ value. In other words, although the utility is larger by taking a greater risk it can be seen that around two-thirds of the time one is worse off. Figure 8 demonstrates clearly to a user, in terms they understand, the consequences and benefits of taking greater risks.

In order to understand the characteristics of this plot, we take a closer look at four levels for the bound relaxation parameter, $\rho$. Figure 9 plots the consumption levels on the tree by their utility. The effect on the consumption levels is visible when the relaxation bound is lowered from 0.8 to 0.7 . During this change the effect from the time-preference criterion for consumption overtakes the effect from the lower bound on consumption, as a 
result consumption in the early periods is higher leaving less money for later consumption. As a consequence, the percentage of nodes that are able to exceed the reference lower bound begins to decrease as the bound relaxation parameter is further decreased.

It is a better choice to also invest in the risky assets with higher expected returns after retirement. Although riskfree investing guarantees a level of consumption, exposing oneself up to an extent to a risky asset brings the opportunity of improving consumption substantially. Interestingly for the utility chosen, the results show that reducing one's guaranteed income below around $80 \%$ of the assured income give little additional benefit.

\section{Conclusions}

We have developed a framework for post-retirement financial planning to obtain the optimal consumption and investment decisions of ones retirement savings during retirement. Our objective was to develop a model with considerable flexibility. One consequence is that this leads to a large nonlinearly constrained optimization problem. However, the problem has considerable structure, which enables its solution by modern optimization methods. One flexibility of particular interest to us is the inclusion of behavioural utilities (and behavioural constraints), which do not satisfy all the axioms of choice. Such utilities are less wellbehaved, making the computation of the optimal consumption and investment decision more challenging. Nonetheless in the framework developed it was shown that such problems were tractable.

In order to keep the model general, we modeled the asset returns using a tree, instead 

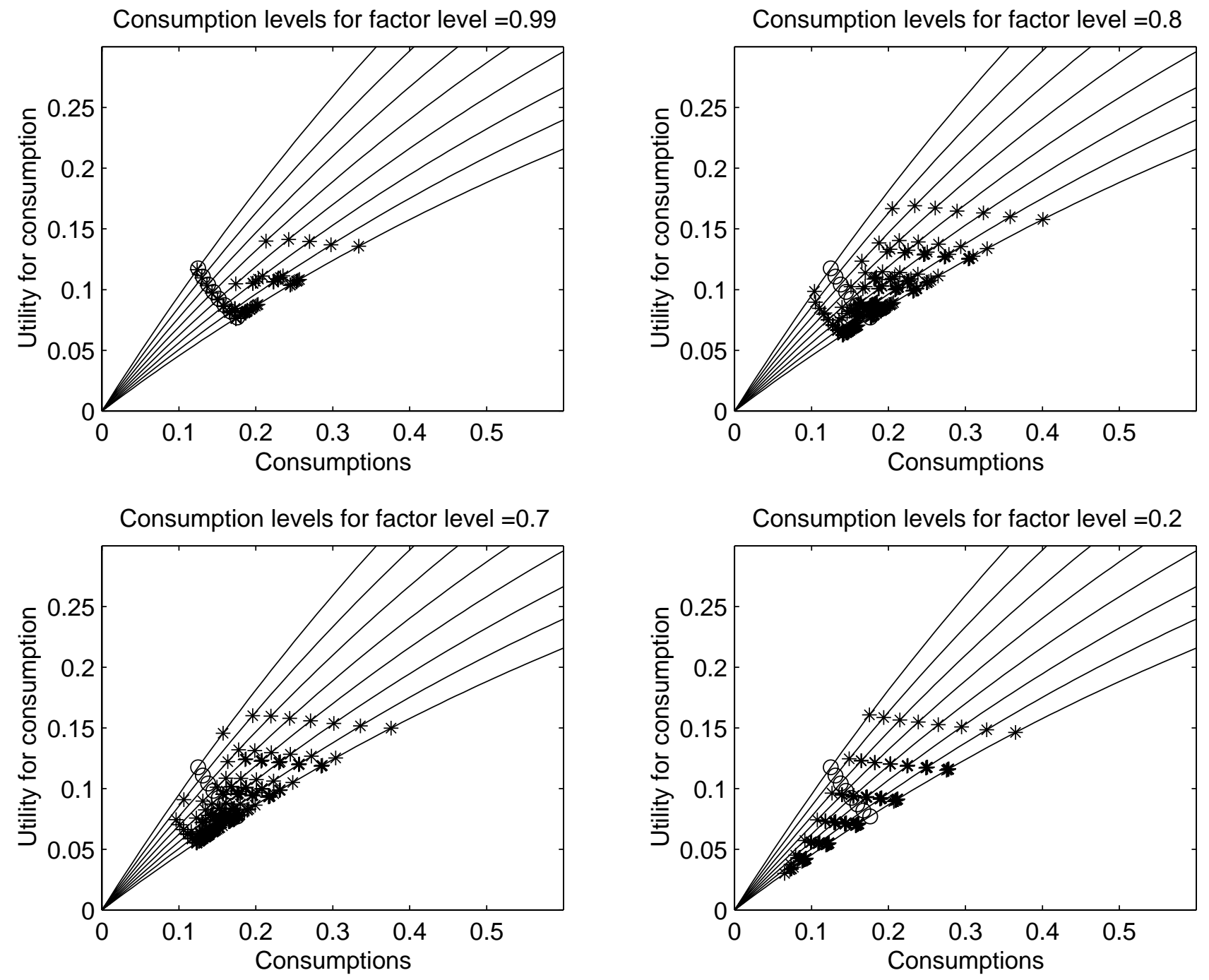

Figure 9: Plot of utility for consumption for 4 levels for the reference lower bound relaxation parameter, relative to the utility for consumption at the reference lower bound level 
of a recombining lattice, even though this implies that the size of the problem increases exponentially with increasing number of time-periods. However, by having non-uniform time-periods the number of periods required can be capped. The choice of a tree structure was essential since the optimal solution obtained for the Prospect theory utility in [11] is wealth-dependent (path-dependent). Therefore, if wealth is treated as a state variable, the tree does not recombine to give a lattice. The problem is framed as a nonlinearly constrained optimization problem. The optimization problem has a structured and sparse constraint Jacobian (this is not the case for a lattice structure) and objective gradients. We use both these characteristics to our computational advantage by using the SNOPT software package. Further, the number of constraints and bounds are comparable to the number of variables, and as a result even though there are several thousand variables in the problem, the number of free variables stays quite manageable. This is another feature that helps improve SNOPT's efficiency.

We solved the problem for the negative exponential utility, a constant absolute risk aversion utility, along with time-preference favouring early consumption. To balance the effect from the time-preference for consumption, we impose lower bounds on consumption, that increase with time. Our particular interest was in studying what, if any, benefit may be obtained from investing in the risky asset. The pre-retirement problem was solved using the Prospect theory loss-aversion utility, which is a behavioural utility function, in Gupta and Murray [11]. Solving the Consumption problem using the Prospect theory utility or other utilities considered in [11] poses no new technical challenges.

We advocate solving the problem with non-uniform time periods. This formulation allows 
for a finer time-refinement for periods at the beginning of the planning period. This feature is particularly attractive when the problem is solved on a rolling basis, since it gives a better resolution on decisions that are immediately implemented, while limiting the growth in the number of variables. The longer periods at the end capture the inherent increased uncertainty farther out in time. It also makes the choice of planning horizon less critical. Another effect of the non-uniform time periods is an enhanced time-preference for consumption. This is because the time discount factor, $\delta$, favours early consumption; and the discounted consumption for a one year period is less than discounted consumption from twelve, one month periods.

\section{Extensions}

We developed a framework for retirement-related financial planning. The retirement-related planning problem is viewed as comprising two subproblems, (i) the problem of acquiring wealth for retiring by saving and optimal investing and (ii) the problem of optimal withdrawals of wealth from the retirement fund after retirement. The retirement savings problem is addressed in Gupta and Murray [11].

The framework developed in this paper for computing the optimal consumption of retirement savings during retirement is quite flexible. We can incorporate multiple asset classes, correlation in the returns of the asset classes, to name a few. Introduction of additional asset classes or correlation in their returns will increase the size of the problem, however the structure and sparsity of the problem will be retained. Additionally, considering transaction costs and tax related issues is also an important extension for the problem. Another exten- 
sion that is trivial to include are constraints, both upper and lower, on consumptions. In the example presented, a lower bound was used that varied in a simple manner with time. This lower bound could be set to any value. Also, upper bounds could be set. By capping consumption, say at come percentage above the reference lower bound, we would likely increase the percentage of time we exceed the reference lower bound. We could also put lower and upper bounds on wealth.

In the solution method we are currently unable to use second derivatives within the optimization algorithm, even though computing these derivatives is possible for our problem. However, algorithms that incorporate second derivatives are under development and will be available shortly. Being able to use this information will give us greater efficiency and ability to solve even larger problems. Another approach to improving efficiency is to use a decomposition algorithm, perhaps in combination with a second-derivative method. The tree structure of the constraints enables the problem to be decomposed (see de Miguel [5]). By using a decomposition approach we replace solving an $n$ variable problem by solving a sequence of $K$ problems with $n / K$ variables, which may be considerably more efficient. A good value for $K$ is $n^{1 / 2}$. Note that the effort to solve a problem usually increases faster than linearly in the size of problem.

To provide a seamless planning tool the pre-retirement planning and post- retirement planning will need to be merged so that there is a smooth transition from one planning phase to the other. The problem will be viewed as having three regions of planning, the first being when the time of retirement is far out, and hence the main focus in on saving and investing. This will be followed by a region where retirement is near and the planner 
will continue to plan the saving and investing for acquiring adequate retirement funds, but also begin to plan the consumption process after retirement. And the third region will be the purely post-retirement planning region when the planner will have retired and will be concerned with the optimal consumption decisions and investment decisions for the remaining wealth in the retirement fund. In the seamless planning framework it may also be desirable to introduce additional stochastic model for health of the planner. This will also lead to better modeling of the planning horizon of the problem.

\section{$7 \quad$ Acknowledgement}

It is a pleasure for us to thank Professor William F. Sharpe for introducing this problem to us and for his encouragement in seeking a solution. We are also indebted to the referees for numerous improvements to the presentation.

\section{References}

[1] David E. Bell, Howard Raiffa, and Amos Tversky. Decision Making - Descriptive, Normative, and Prescriptive Interactions. Cambridge University Press, Cambridge, U.K., 1988.

[2] Zvi Bodie, Robert C. Merton, and William F. Samuelson. Labor supply flexibility and portfolio choice in life-cycle mode. Journal of Economic Dynamics and Control, 16:427-449, 1992. 
[3] John Y. Campbell and Luis M. Viceira. Consumption and portfolio decisions when expected returns are time varying. NBER Working paper No. 5857, 1996.

[4] John Cox, Stephen Ross, and Mark Rubinstein. Option pricing: A simplified approach. Journal of Financial Economics, 7(3):229, 1979.

[5] Angel-Victor DeMiguel. Two decomposition algorithms for optimization problems with coupling variables. PhD thesis, Management Science and Engineering, Stanford University, 2001.

[6] Chi fu Huang and Robert H. Litzenberger. Foundations for Financial Economics. Prentice Hall, Inc., Englewood Cliffs, New Jersy, 1988.

[7] Philip E. Gill, Walter Murray, and Michael A. Saunders. User's Guide for SNOPT(Version 4.0): A Fortran Package for Sparse Nonlinear Programming. Technical Report SOL 86-2, 1998.

[8] Philip E. Gill, Walter Murray, and Michael A. Saunders. Snopt: An sqp algorithm for large-scale constrained optimization. SIAM Journal on Optimization, 12(4):979-1006, 2002.

[9] Philip E. Gill, Walter Murray, and Margaret H. Wright. Practical Optimization. Academic Press, Harcourt Brace and Company, Publishers, London, 1981.

[10] Aparna Gupta. Optimum Asset Allocation with Behavioral Utilities : A Plan for Acquiring and Consuming Retirement Funds. PhD thesis, Scientific Computing and Computational Mathematics Program, Stanford University, 2000. 
[11] Aparna Gupta and Walter Murray. Optimal investment with behavioral utilities using a binomial tree model for asset-returns. Systems Optimization Laboratory Technical Report, 00-1, 2000.

[12] Michael D. Hurd and Katheleen McGarry. The predictive validity of subjective probabilities of survival. NBER Working Paper No 6193, 1997.

[13] Daniel Kahneman and Amos Tversky. Prospect theory: An analysis of decisions under risk. Econometrica, 47:313-327, 1979.

[14] David I. Laibson. Comments on personal retirement saving programs and asset accumulation: Reconciling the evidence. Studies in the Economics of Aging, NBER Project Report, W5599:106-124, 1998.

[15] David G. Luenberger. Investment Science. Oxford University Press, New York, 1998.

[16] Robin L. Lumsdaine, James H. Stock, and Davis A. Wise. Three models of retirement: Computational complexitiy versus predictive validity. NBER Working Paper No 3558, 1990.

[17] Andreu Mas-Colell, Michael D. Winston, and Jerry R. Green. Microeconomic Theory. Oxford University Press, New York, 1995.

[18] Robert C. Merton. Lifetime portfolio selection under uncertainty: The continuous-time case. Review of Economics and Statistics, 51(3):247-257, 1969.

[19] Robert C. Merton. Continuous-Time Finance. Basil Blackwell Inc., Cambridge, Massachusetts $02142,1990$. 
[20] Sheldon M. Ross. Stochastic Processes. John Wiley \& Sons, Inc., New York, 1996.

[21] Mark Rubinstein. Implied binomial trees. The Journal of Finance, 49(3):771-819, 1994.

[22] John Rust. A dynamic programming model of retirement behavior. NBER Working paper No. 2470, 1987.

[23] Paul A. Samuelson. Lifetime portfolio selection by dynamic stochastic programming. Review of Economics and Statistics, 51:239-246, 1969.

[24] William F. Sharpe, Gordon J. Alexander, and Jeffery V. Bailey. Investments. Prentice Hall, Inc. Englewood Cliffs, New Jersey, 1995.

[25] Amos Tversky. Additivity, utility and subjective probability. Journal of Mathematical Psychology, 4:175-202, 1967.

[26] Amos Tversky and Daniel Kahneman. Loss aversion in riskless choice: A referencedependent model. Quarterly Journal of Economics, 106(4):1039-1061, 1991.

[27] Amos Tversky and Daniel Kahneman. Advances in prospect theory: Cumulative representation of uncertainty. Journal of Risk and Uncertainty, 5:297-323, 1992.

[28] Hal R. Varian. Microeconomic Theory. W.W. Norton and Company, Inc., New York, 1992.

[29] Luis M. Viceira. Optimal Consumption and Portfolio Choice for Long-Horizon Investors. PhD thesis, Department of Economics, Harvard University, 1998.

[30] George Wright. Behavioral Decision Making. Plenum Press, New York, N.Y., 1985. 